\title{
Regulation of NMDA-Receptor Synaptic Transmission by Wnt Signaling
}

\author{
Waldo Cerpa, ${ }^{1,2}$ Abigail Gambrill, ${ }^{1}$ Nibaldo C. Inestrosa, ${ }^{2}$ and Andres Barria ${ }^{1}$ \\ ${ }^{1}$ Department of Physiology and Biophysics, University of Washington, Seattle, Washington 98195, and ${ }^{2}$ Centro de Envejecimiento y Regeneración, \\ Departamento de Biología Celular y Molecular, Facultad de Ciencias Biológicas, Pontificia Universidad Católica de Chile, Santiago 8331150, Chile
}

Wnt ligands are secreted glycoproteins controlling gene expression and cytoskeleton reorganization involved in embryonic development of the nervous system. However, their role in later stages of brain development, particularly in the regulation of established synaptic connections, is not known. We found that Wnt-5a acutely and specifically upregulates synaptic NMDAR currents in rat hippocampal slices, facilitating induction of long-term potentiation, a cellular model of learning and memory. This effect requires an increase in postsynaptic $\mathrm{Ca}^{2+}$ and activation of noncanonical downstream effectors of the Wnt signaling pathway. In contrast, Wnt-7a, an activator of the canonical Wnt signaling pathway, has no effect on NMDAR-mediated synaptic transmission. Moreover, endogenous Wnt ligands are necessary to maintain basal NMDAR synaptic transmission, adjusting the threshold for synaptic potentiation. This novel role for Wnt ligands provides a mechanism for Wnt signaling to acutely modulate synaptic plasticity and brain function in later stages of development and in the mature organism.

\section{Introduction}

In the mammalian brain, fast excitatory neurotransmission is mediated mainly by glutamate and its ionotropic receptors AMPAR and NMDAR. Unique properties, including $\mathrm{Ca}^{2+}$ permeability, allow the NMDAR to play a critical role in brain development, neuropathology, and synaptic plasticity, a cellular model of learning and memory (Bliss et al., 2003; Lau and Zukin, 2007; Cline and Haas, 2008). Not surprisingly, the number, properties, and subunit composition of NMDARs present at synapses must be well controlled to regulate calcium influx and different signaling cascades associated with the receptor activation (Yashiro and Philpot, 2008).

Wnt ligands are secreted glycoproteins controlling gene expression and cytoskeleton reorganization with well established roles in the embryonic development of the nervous system of vertebrates and invertebrates. They signal through Frizzled and RoR2 receptors to activate the following: (1) the canonical Wnt/ $\beta$-catenin pathway controlling gene transcription and regulating cytosolic microtubule dynamics; (2) a noncanonical pathway that activates Rho GTPases and c-Jun N-terminal kinase to induce changes in the cytoskeleton; and (3) a noncanonical Wnt/ calcium pathway that increases intracellular $\mathrm{Ca}^{2+}$, activating

\footnotetext{
Received Dec. 3, 2010; revised April 8, 2011; accepted May 7, 2011

Author contributions: W.C., N.C.I., and A.B. designed research; W.C. and A.G. performed research; W.C., A.G., and A.B. analyzed data; A.B. wrote the paper.

This work was supported in part by University of Washington Royalty Research Fund (W.C.) and NIH-NINDS Grant NS060756 (A.B.). We thank Ximena Opitz for technical assistance. This work partially fulfilled the requirements for Waldo Cerpa's doctoral dissertation at the Facultad de Ciencias Biológicas, Pontificia Universidad Católica de Chile.

Correspondence should be addressed to either of the following:Andres Barria, Department of Physiology and Biophysics, Box357290, University of Washington, Seattle, Washington 98195-7290, E-mail: barria@u.washington.edu; or Nibaldo C. Inestrosa, CARE, Departamento de Biología Celular y Molecular, Facultad de Ciencias Biológicas, Pontificia Universidad Católica de Chile, Santiago 8331150, Chile, E-mail: ninestrosa@bio.puc.cl.

D0I:10.1523/JNEUROSCI.6311-10.2011

Copyright $\odot 2011$ the authors $\quad 0270-6474 / 11 / 319466-06 \$ 15.00 / 0$
}

CaMKII and PKC, two enzymes involved in controlling synaptic function and plasticity (Veeman et al., 2003; Kohn and Moon, 2005; Gordon and Nusse, 2006). Wnt proteins participate in processes such as neuronal migration, axon pathfinding, dendritic morphogenesis, and synaptic differentiation (Ciani and Salinas, 2005; Gordon and Nusse, 2006). Dysfunctional Wnt signaling has also been implicated in major neuropathologies including Alzheimer's disease (Zhang et al., 1998) and schizophrenia (Emamian et al., 2004); nonetheless, its normal function in the adult brain is unknown. Recent evidence indicates that Wnt ligands and components of their signaling pathways persist in the adult brain and can modify synaptic function (Inestrosa and Arenas, 2010). A canonical Wnt ligand, Wnt-3a, has been reported to modify a major form of synaptic plasticity, long term potentiation (LTP) (Chen et al., 2006), and Wnt-2 has been shown to induce dendritic arborization (Wayman et al., 2006). Similarly, a noncanonical Wnt ligand, Wnt-5a, has been recently reported to stimulate synaptic differentiation in dissociated cultured neurons in a calcium-dependent manner (Varela-Nallar et al., 2010). However, the mechanisms leading to these acute modifications of established synaptic connections are not well understood.

We tested whether Wnt ligands could acutely regulate glutamatergic synaptic transmission in the Schaffer collateral/commissuralCA1 pathway in rat hippocampal slices. We found that Wnt-5a via $\mathrm{PKC}$ and JNK rapidly potentiates NMDAR currents, facilitating induction of LTP. In addition, we found that endogenous Wnt signaling is necessary for maintenance of NMDAR basal synaptic transmission.

\section{Materials and Methods}

Electrophysiology. Organotypic hippocampal slices were prepared according to standard procedures from postnatal day (P)6 male and female Sprague Dawley rats and maintained in culture for 4-11 d (Opitz-Araya and Barria, 2011). Acute slices were prepared from P17-21 animals 

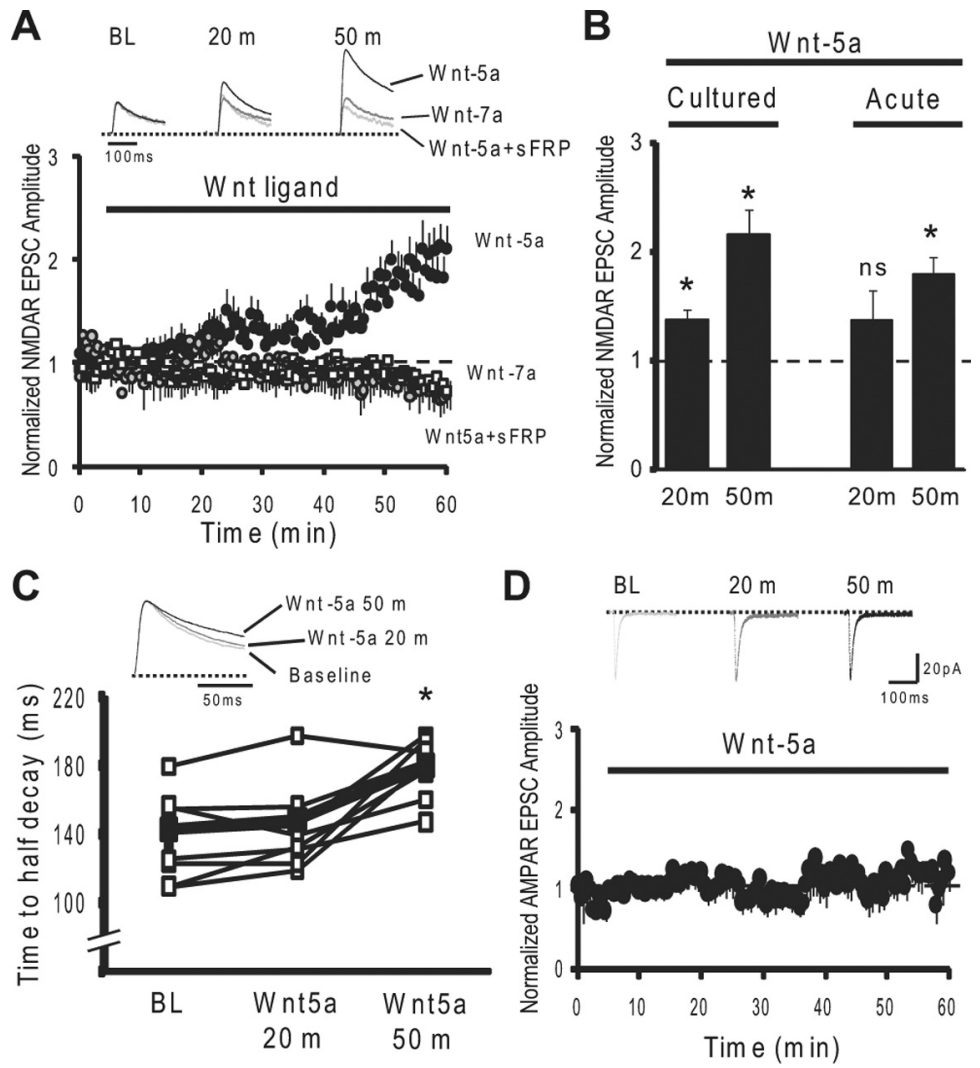

Figure 1. Specific upregulation of NMDAR currents by a noncanonical Wnt-ligand. $A$, Normalized peak amplitude of isolated NMDAR currents recorded at $+40 \mathrm{mV}$. After a period of baseline (BL), conditioned medium containing Wnt- 5 a (black circles; $n=$ 13), Wnt-5a and sFRP (gray circles; $n=3$ ), or Wnt-7a (white squares; $n=5$ ) was added to the bath. Here and in all figures, error bars represent standard error. Inset shows example traces as indicated. $\boldsymbol{B}$, Quantification of the potentiation induced by Wnt- $5 \mathrm{a}$ on the amplitude of NMDAR currents after $20 \mathrm{~min}(n=16)$ and $50 \mathrm{~min}(n=8)$ of treatment in cultured and acute slices $(n=4)$. Asterisk $\left(^{*}\right)$ indicates $p<0.05$ Student's $t$ test compared to baseline. C, Time to half-decay of NMDAR EPSCs during baseline and after 20 and 50 min of Wnt- 5 a treatment for each cell. Average in bold $(n=8)$. Asterisk indicates $p<0.05$ Student's $t$ test compared to baseline and $20 \mathrm{~min}$. Inset shows representative traces of normalized NMDAR EPSCs to visualize differences in the decay kinetics. $\boldsymbol{D}$, Normalized peak amplitude of AMPAR EPSCS. After a period of baseline, conditioned medium containing Wnt-5a was added to the bath $(n=4)$. Inset shows representative traces as indicated.

(Gambrill et al., 2011). Animals were handled in accordance with University of Washington (Seattle, WA) guidelines. Whole-cell recordings of CA1 pyramidal cells were obtained under visual guidance. The recording chamber was constantly perfused with artificial CSF (ACSF) containing $119 \mathrm{~mm} \mathrm{NaCl}, 2.5 \mathrm{~mm} \mathrm{KCl}, 4 \mathrm{~mm} \mathrm{CaCl}_{2}, 4 \mathrm{~mm} \mathrm{MgCl}_{2}, 26 \mathrm{~mm} \mathrm{NaHCO}_{3}$, $1 \mathrm{~mm} \mathrm{NaH} \mathrm{PO}_{4}, 11 \mathrm{~mm}$ glucose, $0.1 \mathrm{~mm}$ picrotoxin, $2 \mu \mathrm{M}$ 2-chloroadenosine, $\mathrm{pH} 7.4$, and gassed with $5 \% \mathrm{CO}_{2} / 95 \% \mathrm{O}_{2}$. Recordings were made at room temperature $\left(22-24^{\circ} \mathrm{C}\right)$. Patch recording pipettes $(\sim 4 \mathrm{M} \Omega)$ were filled with intracellular solution containing 115 mM cesium methanesulfonate, $20 \mathrm{~mm} \mathrm{CsCl}, 10 \mathrm{~mm}$ HEPES, $2.5 \mathrm{~mm}$ $\mathrm{MgCl}_{2}, 4 \mathrm{~mm} \mathrm{Na}_{2} \mathrm{ATP}, 0.4 \mathrm{~mm} \mathrm{Na}_{3} \mathrm{GTP}, 10 \mathrm{~mm}$ sodium phosphocreatine, and 0.6 mM EGTA (pH 7.25). Synaptic responses were evoked by a bipolar electrode placed over Schaffer collateral fibers $\sim 200-400 \mu \mathrm{m}$ from the targeted CA1 cell. Evoked NMDAR responses were recorded at a holding potential of $+40 \mathrm{mV}$ in the presence of NBQX ( $2 \mu \mathrm{M})$. Spontaneous miniature events (mEPSCs) were recorded at a holding potential of $-60 \mathrm{mV}$ in the presence of TTX $(1 \mu \mathrm{M})$. Conditioned medium containing Wnt proteins was obtained by expression of the desired Wnt protein in HEK-293 as reported previously (Hall et al., 2000) and bath applied.

Imaging. Organotypic hippocampal slices were transfected with EGFP using biolistics (Woods and Zito, 2008). Transfected CA1 pyramidal neurons were live imaged on an Olympus FV confocal laser scanning microscope at $32-34^{\circ} \mathrm{C}$. Dendrites were imaged with $0.5 \mu \mathrm{M}$ steps in the $z$-axis. Each optical plane was averaged two or three times (Kalman filter). Images were analyzed with ImageJ (NIH, version 1.4). Spines were defined as protrusions from the dendrite $<3$ $\mu \mathrm{m}$ long and with a round head.

Statistics. Kolmogorov-Smirnoff test was used to analyze cumulative distribution of the inter-event interval. Bonferroni's multiplecomparison test was used to compare the level of potentiation among cells treated with several kinase inhibitors and untreated control cells. All other analysis used mean \pm SEM and Student's $t$ test.

\section{Results \\ Potentiation of NMDAR-mediated currents by Wnt ligands}

We recorded isolated EPSCs mediated by NMDARs or AMPARs in cultured organotypic hippocampal slices. After a $5 \mathrm{~min}$ baseline period, we bath applied conditioned medium containing various Wnt ligands. Wnt-5a, a noncanonical ligand (Gordon and Nusse, 2006), produced a two-step increase in the amplitude of NMDAR responses, suggesting two different mechanisms involved in the potentiation of NMDAR function. In contrast, application of Wnt-7a, a canonical Wnt ligand, did not affect NMDAR currents (Fig. 1A). In cultured slices, Wnt-5a increased NMDAR currents significantly after $20 \mathrm{~min}$ (40\% over baseline) and twofold after $50 \mathrm{~min}$ (Fig. $1 \mathrm{~B}$, left). Similarly, in acute slices $20 \mathrm{~min}$ of Wnt-5a treatment produced a $37 \%$ increase that was not statistically significant; this increased to $80 \%$, which was statistically significant, after $50 \mathrm{~min}$ of treatment (Fig. $1 B$, right). Control experiments with heat-inactivated conditioned medium or conditioned medium from cells expressing empty vector produced no potentiation (data not shown). Simultaneous application of Wnt-5a and the secreted frizzled-related protein 1 (sFRP1) prevented potentiation of NMDAR currents (Fig. 1A). Physical interaction between sFRP1 and Wnt-5a allows sFRP1 to act as an effective Wnt scavenger that can inhibit Wnt-5a signaling (Rattner et al., 1997; Matsuyama et al., 2009).

Following Wnt-5a-induced potentiation, NMDAR currents became significantly slower after $50 \mathrm{~min}$ compared to responses before treatment. Also, responses after $50 \mathrm{~min}$ are significantly slower that responses after 20 min of treatment (Fig. 1C). Kinetics of NMDARs are determined by the NR2 subunit, with NR2Bcontaining receptors exhibiting slower decaying currents than NR2A-containing receptors (Traynelis et al., 2010). Using Ro256981, a noncompetitive NR2B selective blocker (Fischer et al., 1997), we confirmed that Wnt-5a increases the proportion of synaptic NR2B-containing receptors. In neurons from control untreated slices, $1 \mu \mathrm{M}$ Ro25-6981 blocked 46\% \pm 8.04 of NMDAR EPSCs $(n=5)$. In contrast, in neurons treated with Wnt-5a for $1 \mathrm{~h}$, Ro25-6981 blocked $73 \% \pm 6.05$ of NMDAR currents $(n=3 ; p<0.05$ compared to control cells). These experiments suggest that Wnt-5a increases the synaptic content of NR2B-containing receptors.

Importantly, the effect of Wnt-5a on glutamatergic synaptic transmission is specific for NMDAR-mediated currents. Bath ap- 
plication of Wnt-5a did not affect AMPAR-mediated EPSCs (Fig. 1D). The specificity of the effect of Wnt-5a on NMDAR-mediated transmission suggests that Wnt-5a is not altering the presynaptic glutamate release machinery. To confirm this, we examined whether the probability of glutamate release changes after Wnt-5a treatment of the slice. Paired pulse facilitation of AMPAR-mediated EPSCs was not different before or after 20 or $50 \mathrm{~min}$ of Wnt-5a treatment (Fig. 2A). We also determined the frequency of AMPARmediated spontaneous mEPSCs and the density of dendritic spines as measurements of number of synapses (Gambrill and Barria, 2011). Neither the frequency nor the amplitude of AMPAR-mediated mEPSCs changed after 45-55 min of Wnt-5a treatment compared to the baseline period before treatment (Fig. $2 B$ ). In addition, spine density in CA1 pyramidal neurons did not change before and after a $1 \mathrm{~h} \mathrm{Wnt-5a}$ treatment (Fig. 2C,D).

Together, these results indicate that Wnt-5a neither alters presynaptic function nor increases the number of synaptic contacts. However, Wnt-5a can potentiate NMDAR function within $20 \mathrm{~min}$ and increase the proportion of NR2Bcontaining NMDARs present at the synapse.

\section{PKC and JNK are biochemical mediators of NMDAR potentiation}

To investigate the signaling pathways involved in the upregulation of NMDAR function, we delivered a calcium chelator or specific protein kinase inhibitors via the recording pipette. This allowed us to target potential postsynaptic elements of the noncanonical pathway activated by Wnt-5a that could regulate NMDAR function.

First we monitored NMDAR-mediated synaptic transmission with a recording pipette containing BAPTA, a calcium chelator. After a 5 min baseline period, Wnt-5a was bath applied as in Figure 1. The presence of BAPTA in the pipette completely eliminated the potentiation of NMDAR currents induced by Wnt-5a (Fig. $3 A, D$ ). Next, we targeted PKC and JNK, two known downstream signaling kinases of the noncanonical pathway (Ciani and Salinas, 2005). Inhibition of $\mathrm{Ca}^{2+}$-dependent $\mathrm{PKC}$ isoforms with Go6976 or the more general PKC inhibitor calphostin C eliminated the potentiation of NMDAR currents at $20 \mathrm{~min}$ compared to their baseline (Fig. $3 B, D$ ). However, inhibition of PKC left intact the slow increase in NMDAR current amplitude that developed over $50 \mathrm{~min}$, producing a significant potentiation of NMDAR currents over their baseline (Fig. $3 B, D$ ). The level of potentiation after $50 \mathrm{~min}$ in the presence of PKC inhibitors is not significantly different from the level of potentiation in control cells ( $p<0.05$ Bonferroni's multiple-comparison test).

The slower developing increase in NMDAR currents was blocked by two different JNK inhibitors, TI-JIP153-163 and SP600125. However, inhibition of JNK did not block the early PKC-dependent potentiation. TI-JIP153-163 allowed a significant $34 \%$ potentiation at $20 \mathrm{~min}$ and SP600125 allowed a nonsignificant potentiation of $18 \%$ compared to baseline (Fig. 3C,D). This level of
B

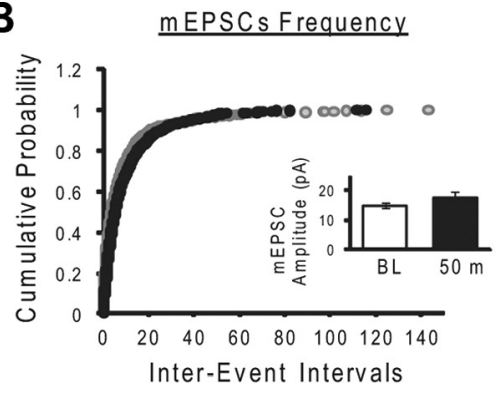

D

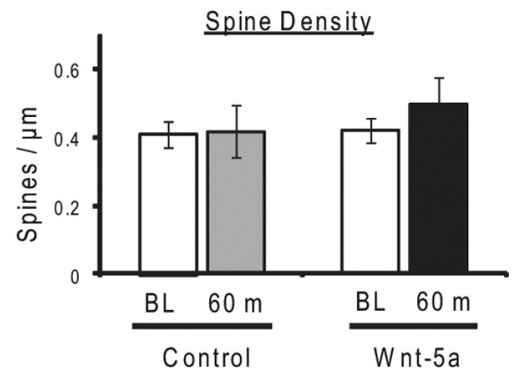

Figure 2. Wnt-5a does not increase the number of synapses. $\boldsymbol{A}$, Paired-pulse facilitation (peak2-peak1)/peak1) measured baseline period (gray circles) or a 10 min period after 50 min of adding Wnt-5a to the bath (black circles; $n=5$ ). Inset shows ing acquired from cultured slices before (baseline) or after $50 \mathrm{~min}$ of adding conditioned medium from cells expressslices treated as indicated. Control, $n=3$; Wnt-5a-treated slices, $n=3$.

potentiation in the presence of these two inhibitors was not different from the level of potentiation in control cells (Fig. $3 D ; p<0.05$ Bonferroni's multiple comparison test). Interestingly, this potentiation at 20 min was transient and NMDAR currents returned to baseline values after $50 \mathrm{~min}$ (Fig. 3C,D).

These experiments indicate that Wnt-5a triggers a transient PKC-dependent potentiation of NMDAR currents and a slower JNK-dependent potentiation that does not require previous activation of PKC, suggesting independent mechanisms.

\section{Endogenous Wnt ligands regulate basal NMDAR-dependent} synaptic transmission

To investigate the physiological relevance of NMDAR regulation by the noncanonical Wnt pathway, we tested whether endogenous Wnt regulates glutamatergic transmission. We monitored NMDAR-mediated synaptic transmission and, after a 5 min baseline period, bath applied the Wnt scavenger sFRP1. Removing endogenous Wnt via application of sFRP1 acutely decreased the amplitude of NMDAR currents (Fig. 4A). AMPAR-mediated EPSCs were not affected by sFRP1 treatment (Fig. $4 B$ ), indicating that endogenous Wnt specifically regulates NMDAR function. After treatment of the slices with sFRP1, the decay kinetics of NMDAR EPSCs did not change significantly; however, a trend toward faster kinetics was observed (Fig. 4C). Similarly, sensitivity to $1 \mu \mathrm{M}$ Ro25-6982 did not change significantly, but the blockade of NMDAR currents is reduced from $48 \% \pm 1.44$ in control cells to $44 \% \pm 2.03$ in cells treated with sFRP1 for $1 \mathrm{~h}$. This suggests that sFRP1 either affects NR2A and NR2B equally, or that the change in NR2B/NR2A ratio is small and not detected by these techniques.

If a continuous Wnt signal is necessary to maintain NMDAR basal transmission, blockade of downstream signaling should also diminish basal NMDAR transmission. We monitored basal 
A

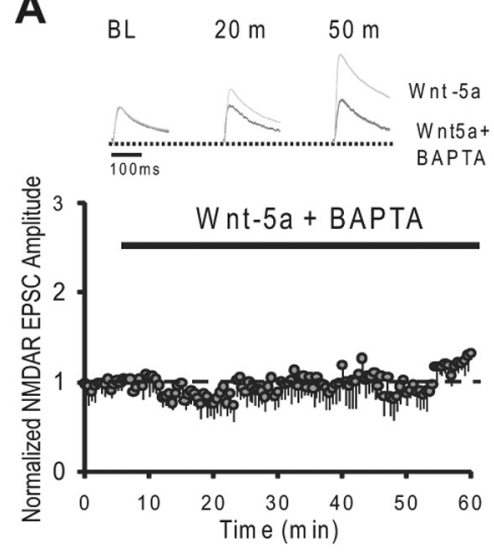

B

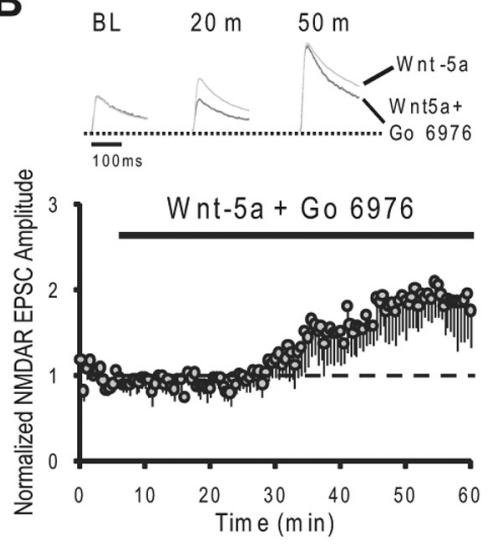

C

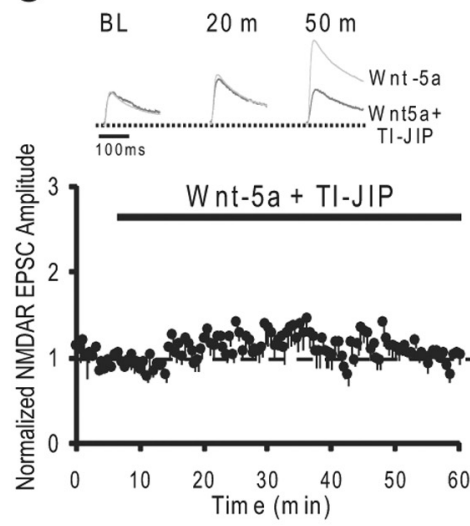

D

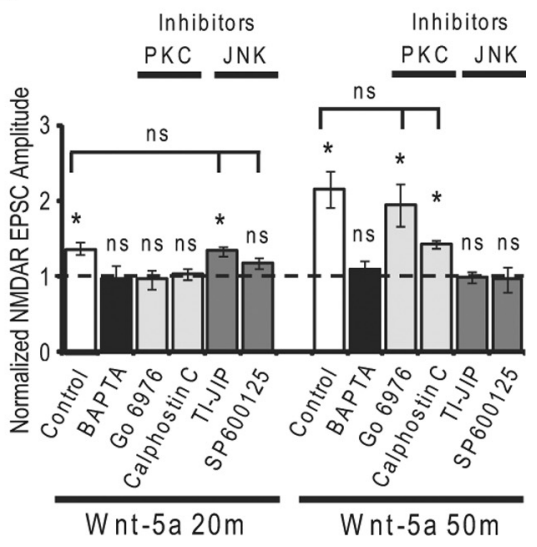

Figure 3. Wnt-5a upregulation of NMDAR currents requires $\mathrm{Ca}^{+2}$ and activation of PKC and JNK. $A$, Normalized peak amplitude of isolated NMDAR currents recorded at $+40 \mathrm{mV}$ with internal solution containing $10 \mathrm{~mm}$ BAPTA $(n=4)$. After baseline (BL), conditioned medium containing Wnt-5a was added to the bath. Insets show representative traces as indicated. Traces from control cells (no inhibitors in the pipette) are superimposed for comparison. $\boldsymbol{B}$, Normalized peak amplitude of isolated NMDAR currents recorded at $+40 \mathrm{mV}$ with internal solution containing $200 \mathrm{~nm}$ G06976 $(n=4)$. Insets are as in $A$. C, Normalized peak amplitude of isolated NMDAR currents recorded at $+40 \mathrm{mV}$ with internal solution containing $1.7 \mu \mathrm{M}$ TI-JIP153-163 $(n=4)$. Insets are as in $\boldsymbol{A}$. D, Quantification of the potentiation induced by Wnt-5a on the amplitude of NMDAR currents after 20 or 50 min of treatment in control cells or cells recorded in the presence of BAPTA, PKC inhibitors, or JNK inhibitors. Asterisk $\left(^{*}\right)$ indicates $p<0.05$ Student's $t$ test compared to baseline.

NMDAR synaptic transmission with recording pipettes containing PKC or JNK inhibitors. We normalized the peak amplitudes to the peak values obtained shortly after acquiring wholecell configuration before the inhibitors dialyze into the cell. Delivery of a PKC inhibitor via the recording pipette diminishes basal NMDAR transmission. Blockade of JNK also caused a small decrease in basal NMDAR transmission (Fig. $4 D$ ).

Our results indicate that endogenous Wnt ligands are present in the slice and are required for the maintenance of basal NMDAR synaptic transmission.

Regulation of NMDAR synaptic transmission by Wnt ligands affects the threshold for LTP induction

The level of postsynaptic responses required to induce plasticity and determine its direction, the threshold function, varies as a function of the history of synaptic activity (Abraham, 2008) and depends on the level of NMDAR function (Cummings et al., 1996). Wnt-5a increases NMDAR currents and the NR2B/NR2A synaptic ratio. This change in subunit composition changes the charge transfer and kinetics of synaptic NMDAR EPSCs (Traynelis et al., 2010) as well as the interaction with CaMKII, an interaction required for LTP (Barria and Malinow, 2005). These changes, in turn, would be expected to lower the activity requirements for evoking LTP. Conversely, a decrease in NMDAR synaptic transmission induced by sFRP1 should increase the activity requirements for LTP induction. To test whether Wnt signaling modulates the threshold for plasticity, we induced LTP using a common protocol that pairs presynaptic activity and postsynaptic depolarization (Malinow and Tsien, 1990). We induced LTP in control slices or slices pretreated for $1 \mathrm{~h}$ with Wnt-5a or sFRP1 (Fig. $4 E$ ). The level of potentiation after 25-30 min was significantly larger in cultured or acute slices pretreated with Wnt-5a compared to control slices (Fig. $4 E, F$ ). On the other hand, the level of potentiation in slices pretreated with sFRP1 was significantly smaller compared to control slices (Fig. $4 E, F$ ).

Thus, Wnt ligands can regulate the induction of synaptic plasticity via rapid regulation of NMDAR currents and subunit composition of synaptic NMDARs.

\section{Discussion}

Rapid and specific modulation of NMDAR currents by Wnt-5a in hippocampal slices is a novel role for Wnt ligands that are normally associated with embryonic development and maturation of the nervous system.

Our experiments identify postsynaptic PKC and JNK as biochemical mediators of NMDAR potentiation. It has been shown that PKC increases the channel opening rate (Lan et al., 2001) and surface expression of NMDARs, the later via phosphorylation of SNAP-25, a membrane fusion protein of the SNARE family (Lau et al., 2010). Interestingly, the PKC-dependent potentiation that occurs within the first 20 min returns to basal levels if JNK is inhibited. This suggests that JNK is necessary to stabilize the effects induced by PKC. JNK has been shown to slowly rearrange postsynaptic components, such as PSD95, necessary to anchor NMDARs at synaptic sites (Farías et al., 2009). The JNK pathway is also involved in cytoskeleton dynamics regulating the phosphorylation state of microtubule-associated proteins such as MAP-2 (Björkblom et al., 2005). During the time window of our experiments, we did not find evidence of structural changes such as changes in dendritic spine morphology or density, in contrast to what has been observed in dissociated cultured neurons (Varela-Nallar et al., 2010). Different preparations presumably account for the difference. Our data in hippocampal slices argue in favor of local and acute modification of proteins such as PSD-95, which is phosphorylated by JNK, increasing its synaptic stability (Kim et al., 2007), but do not rule out a more general and slower cell-wide effect that could involve remodeling of synaptic and/or dendritic structures.

A previous report indicates that Wnt-5a also increases AMPAR-mediated EPSCs (Cerpa et al., 2010). We did not observe any effect of Wnt-5a on AMPAR currents in cultured or 
acute slices, ruling out a difference due to different preparations. We suggest that the discrepancy arises because in the previous study, stimulation of the afferents was interrupted after acquiring a baseline. It has been reported that a long period of no stimulation after baseline ( $>20 \mathrm{~min}$ ) induces homeostatic potentiation of NMDAR as well as AMPAR currents (Gambrill et al., 2011).

It is important to note that endogenous Wnt ligands are present in the hippocampus and that their activity is necessary to maintain basal NMDAR synaptic transmission. Since blockade of Wnt-5a downstream signaling cascades causes a similar effect as sequestering endogenous Wnt ligands, we conclude that endogenous noncanonical ligands regulate basal NMDAR synaptic transmission.

Modification of NMDAR function is thought to underlie dynamic adjustment of the threshold for synaptic plasticity, a necessary mechanism for proper information storage and plasticity of sensory circuits (Abraham, 2008; Smith et al., 2009). Wnt signaling can acutely and specifically modulate NMDAR currents, contributing to adjust the threshold for synaptic plasticity.

Regulation of basal synaptic transmission and synaptic plasticity provides a mechanism for Wnt signaling to modulate brain functions acutely. This mechanism could explain the role of dysfunctional Wnt signaling in neuropathologies where the NMDAR function is compromised.

\section{References}

Abraham WC (2008) Metaplasticity: tuning synapses and networks for plasticity. Nat Rev Neurosci 9:387.

Barria A, Malinow R (2005) NMDA receptor subunit composition controls synaptic plasticity by regulating binding to CaMKII. Neuron 48:289-301.

Björkblom B, Ostman N, Hongisto V, Komarovski V, Filén JJ, Nyman TA, Kallunki T, Courtney MJ, Coffey ET (2005) Constitutively active cytoplasmic c-Jun $\mathrm{N}$-terminal kinase 1 is a dominant regulator of dendritic architecture: role of microtubule-associated protein 2 as an effector. J Neurosci 25:6350-6361.

Bliss TV, Collingridge GL, Morris RG (2003) Introduction. Long-term potentiation and structure of the issue. Philos Trans R Soc Lond B Biol Sci 358:607-611.

Cerpa W, Farías GG, Godoy JA, Fuenzalida M, Bonansco C, Inestrosa NC (2010) Wnt-5a occludes Abeta oligomer-induced depression of glutamatergic transmission in hippocampal neurons. Mol Neurodegener 5:3.

Chen J, Park CS, Tang SJ (2006) Activity-dependent synaptic Wnt release regulates hippocampal long term potentiation. J Biol Chem 281:11910-11916.

Ciani L, Salinas PC (2005) WNTs in the vertebrate nervous system: from patterning to neuronal connectivity. Nat Rev Neurosci 6:351-362.

Cline H, Haas K (2008) The regulation of dendritic arbor development and plasticity by glutamatergic synaptic input: a review of the synaptotrophic hypothesis. J Physiol 586:1509-1517.
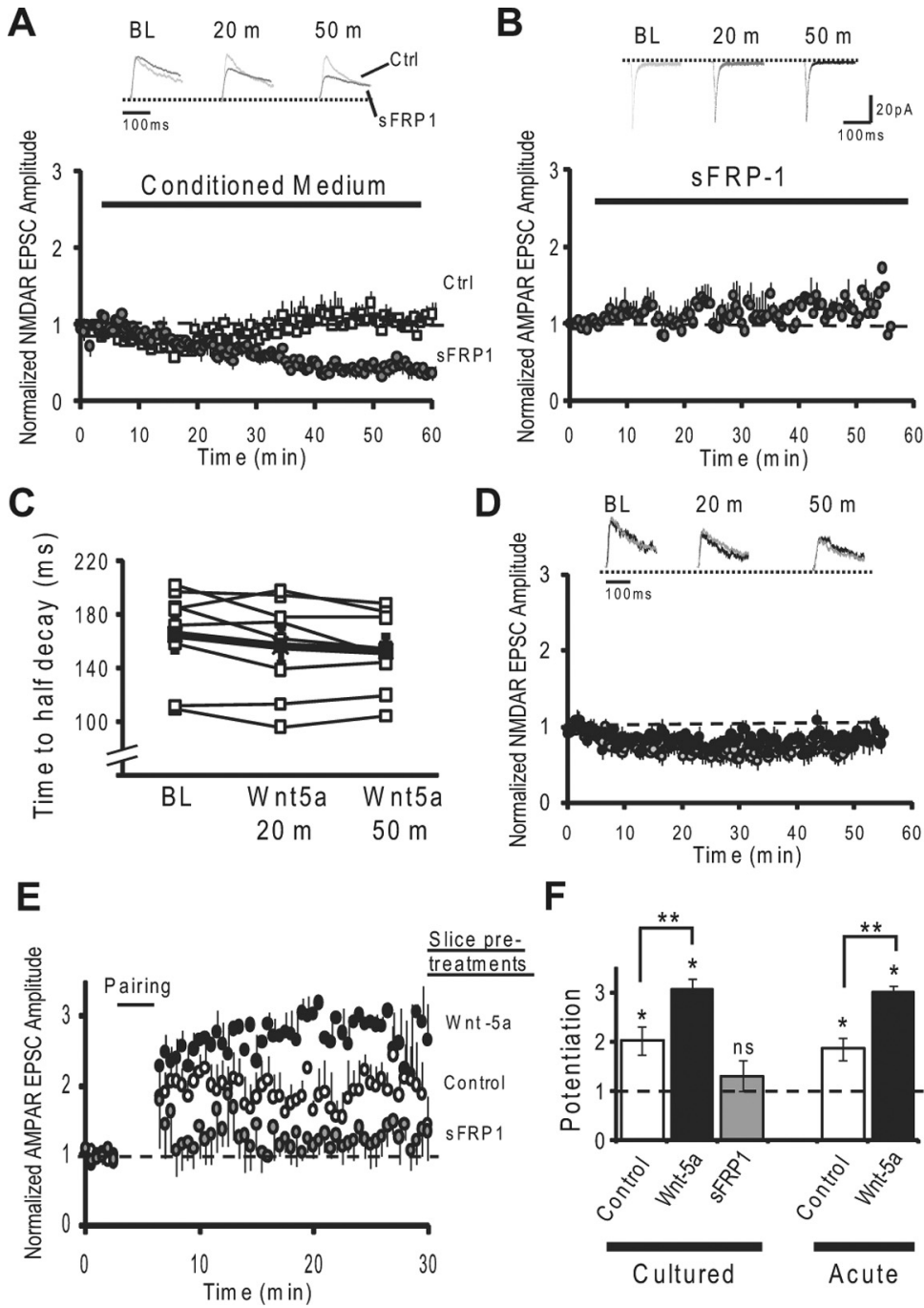

Figure 4. Wnt signaling is necessary to maintain NMDAR basal transmission and modulates synaptic plasticity. $A$, Normalized peak amplitude of isolated NMDAR currents recorded at $+40 \mathrm{mV}$. After baseline (BL) period, conditioned medium from cells expressing an empty vector $(n=4)$ or cells expressing the Wnt scavenger sFRP1 $(n=6)$ were added to the bath. Inset shows r sFRP was added to the bath $(n=4)$. Inset shows representative traces as indicated. $C$, Time to half-decay of NMDAR EPSCs NMDAR currents were recorded with internal solution containing $200 \mathrm{~nm}$ G06976 $(n=7)$ or $1.7 \mu \mathrm{M}$ 7). Peak amplitudes were normalized to the average amplitude of the first 3 min of recording. Inset shows slices $(n=7)$, slices treated for 40 min with Wnt-5a $(n=4)$, or Wnt scavenger SFP1 $(n=4)$. After treating slices, whole tion was obtained and, after a short baseline period, LTP was induced by pairing $3 \mathrm{~Hz}$ stimulation with $0 \mathrm{mV}$ depolarization for $1.5 \mathrm{~min} . \boldsymbol{F}$, Quantification of the potentiation induced by pairing in cultured as well as in acute slices treated as indicated. ${ }^{*} p<0.05$, Student's $t$ test compared to baseline; ${ }^{* *} p<0.05$, Bonferroni's multiple-comparison test.

Cummings JA, Mulkey RM, Nicoll RA, Malenka RC (1996) $\mathrm{Ca}^{2+}$ signaling requirements for long-term depression in the hippocampus. Neuron 16:825-833.

Emamian ES, Hall D, Birnbaum MJ, Karayiorgou M, Gogos JA (2004) Convergent evidence for impaired AKT1-GSK3beta signaling in schizophrenia. Nat Genet 36:131-137.

Farías GG, Alfaro IE, Cerpa W, Grabowski CP, Godoy JA, Bonansco C, Inestrosa NC (2009) Wnt-5a/JNK signaling promotes the clustering of PSD-95 in hippocampal neurons. J Biol Chem 284:15857-15866.

Fischer G, Mutel V, Trube G, Malherbe P, Kew JN, Mohacsi E, Heitz MP, Kemp JA (1997) Ro 25-6981, a highly potent and selective blocker of $\mathrm{N}$-methyl-D-aspartate receptors containing the NR2B subunit: characterization in vitro. J Pharmacol Exp Ther 283:1285-1292.

Gambrill AC, Barria A (2011) NMDA receptor subunit composition con- 
trols synaptogenesis and synapse stabilization. Proc Natl Acad Sci U S A 108:5855-5860

Gambrill AC, Storey GP, Barria A (2011) Dynamic regulation of NMDA receptor transmission. J Neurophysiol 105:162-171.

Gordon MD, Nusse R (2006) Wnt signaling: multiple pathways, multiple receptors, and multiple transcription factors. J Biol Chem 281:22429-22433.

Hall AC, Lucas FR, Salinas PC (2000) Axonal remodeling and synaptic differentiation in the cerebellum is regulated by WNT-7a signaling. Cell 100:525-535.

Inestrosa NC, Arenas E (2010) Emerging roles of Wnts in the adult nervous system. Nat Rev Neurosci 11:77-86.

Kim MJ, Futai K, Jo J, Hayashi Y, Cho K, Sheng M (2007) Synaptic accumulation of PSD-95 and synaptic function regulated by phosphorylation of serine-295 of PSD-95. Neuron 56:488-502.

Kohn AD, Moon RT (2005) Wnt and calcium signaling: beta-cateninindependent pathways. Cell Calcium 38:439-446.

Lan JY, Skeberdis VA, Jover T, Grooms SY, Lin Y, Araneda RC, Zheng X, Bennett MV, Zukin RS (2001) Protein kinase C modulates NMDA receptor trafficking and gating. Nat Neurosci 4:382-390.

Lau CG, Zukin RS (2007) NMDA receptor trafficking in synaptic plasticity and neuropsychiatric disorders. Nat Rev Neurosci 8:413-426.

Lau CG, Takayasu Y, Rodenas-Ruano A, Paternain AV, Lerma J, Bennett MV, Zukin RS (2010) SNAP-25 is a target of protein kinase C phosphorylation critical to NMDA receptor trafficking. J Neurosci 30:242-254.

Malinow R, Tsien RW (1990) Presynaptic enhancement shown by wholecell recordings of long-term potentiation in hippocampal slices. Nature 346:177-180.

Matsuyama M, Aizawa S, Shimono A (2009) Sfrp controls apicobasal polarity and oriented cell division in developing gut epithelium. PLoS Genet 5:e1000427.

Opitz-Araya X, Barria A (2011) Organotypic hippocampal slice cultures. J Vis $\operatorname{Exp} 48: \mathrm{e} 2462$.
Rattner A, Hsieh JC, Smallwood PM, Gilbert DJ, Copeland NG, Jenkins NA, Nathans J (1997) A family of secreted proteins contains homology to the cysteine-rich ligand-binding domain of frizzled receptors. Proc Natl Acad Sci U S A 94:2859-2863.

Smith GB, Heynen AJ, Bear MF (2009) Bidirectional synaptic mechanisms of ocular dominance plasticity in visual cortex. Philos Trans R Soc Lond B Biol Sci 364:357-367.

Traynelis SF, Wollmuth LP, McBain CJ, Menniti FS, Vance KM, Ogden KK, Hansen KB, Yuan H, Myers SJ, Dingledine R (2010) Glutamate receptor ion channels: structure, regulation, and function. Pharmacol Rev 62:405-496.

Varela-Nallar L, Alfaro IE, Serrano FG, Parodi J, Inestrosa NC (2010) Wingless-type family member $5 \mathrm{~A}$ (Wnt-5a) stimulates synaptic differentiation and function of glutamatergic synapses. Proc Natl Acad Sci U S A 107:21164-21169.

Veeman MT, Axelrod JD, Moon RT (2003) A second canon: functions and mechanisms of beta-catenin-independent Wnt signaling. Dev Cell 5:367-377.

Wayman GA, Impey S, Marks D, Saneyoshi T, Grant WF, Derkach V, Soderling TR (2006) Activity-dependent dendritic arborization mediated by CaM-kinase I activation and enhanced CREB-dependent transcription of Wnt-2. Neuron 50:897-909.

Woods G, Zito K (2008) Preparation of gene gun bullets and biolistic transfection of neurons in slice culture. J Vis Exp 12:e675.

Yashiro K, Philpot BD (2008) Regulation of NMDA receptor subunit expression and its implications for LTD, LTP, and metaplasticity. Neuropharmacology 55:1081-1094.

Zhang Z, Hartmann H, Do VM, Abramowski D, Sturchler-Pierrat C, Staufenbiel M, Sommer B, van de Wetering M, Clevers H, Saftig P, De Strooper B, He X, Yankner BA (1998) Destabilization of beta-catenin by mutations in presenilin-1 potentiates neuronal apoptosis. Nature 395:698-702. 